University of Nebraska - Lincoln DigitalCommons@University of Nebraska - Lincoln

Public Health Resources

Public Health Resources

2012

\title{
Vaccination Coverage Among American Indian and Alaska Native Children, 2006-2010
}

Amy V. Groom

Centers for Disease Control and Prevention, Atlanta, Georgia

Tammy A. Santibanez

Centers for Disease Control and Prevention, Atlanta, Georgia

Ralph T. Bryan

Centers for Disease Control and Prevention, Atlanta, Georgia

Follow this and additional works at: http://digitalcommons.unl.edu/publichealthresources

Groom, Amy V.; Santibanez, Tammy A.; and Bryan, Ralph T., "Vaccination Coverage Among American Indian and Alaska Native Children, 2006-2010" (2012). Public Health Resources. 436.

http://digitalcommons.unl.edu/publichealthresources/436

This Article is brought to you for free and open access by the Public Health Resources at DigitalCommons@University of Nebraska - Lincoln. It has been accepted for inclusion in Public Health Resources by an authorized administrator of DigitalCommons@University of Nebraska - Lincoln. 


\section{Vaccination Coverage Among American Indian and Alaska Native Children, 2006-2010}

WHAT'S KNOWN ON THIS SUBJECT: Disparities in vaccination coverage between American Indian/Alaska Native and white children previously existed between 2001 and 2004 but were not present in 2005 .

WHAT THIS STUDY ADDS: This study updates a previous study by analyzing data through 2010 and found that these gains have been maintained.

\section{abstract}

BACKGROUND AND OBJECTIVES: A previous study on vaccination coverage in the American Indian/Alaska Native (AI/AN) population found that disparities in coverage between Al/AN and white children existed from 2001 to 2004 but were absent in 2005. The objective of this study was to describe vaccination coverage levels for Al/AN children aged 1935 months in the United States between 2006 and 2010, examining whether gains found for Al/AN children in 2005 have been sustained.

METHODS: Data from the 2006 through 2010 National Immunization Surveys were analyzed. Groups were defined as Al/AN (alone or in combination with any other race and excluding Hispanics) and white-only non-Hispanic children. Comparisons in demographics and vaccination coverage were made.

RESULTS: Demographic risk factors often associated with underimmunization were significantly higher for Al/AN respondents compared with white respondents in most years studied. Overall, vaccination coverage was similar between the 2 groups in most years, although coverage with 4 or more doses of pneumococcal conjugate vaccine was lower for Al/AN children in 2008 and 2009, as was coverage with vaccine series measures the series in 2006 and 2009. When stratified by geographic regions, Al/AN children had coverage that was similar to or higher than that of white children for most vaccines in most years studied.

CONCLUSIONS: The gains in vaccination coverage found in 2005 have been maintained. The absence of disparities in coverage with most vaccines between Al/AN children and white children from 2006 through 2010 is a clear success. These types of periodic reviews are important to ensure we remain vigilant. Pediatrics 2012;130: e1592-e1599
AUTHORS: Amy V. Groom, MPH, ${ }^{\mathrm{a}, \mathrm{b}}$ Tammy A. Santibanez, $\mathrm{PhD}^{\mathrm{a}}$ and Ralph T. Bryan, $\mathrm{MD}^{\mathrm{c}}$

almmunization Services Division, National Center for Immunization and Respiratory Diseases, Centers for Disease Control and Prevention, Atlanta, Georgia; ${ }^{b}$ Division of Epidemiology and Disease Prevention, Office of Public Health Support, Indian Health Service, Albuquerque, New Mexico; and cIndependent Consultant. Formerly with Office of Minority Health and Health Disparities, Office of Strategy and Innovation, Office of the Director, Centers for Disease Control and Prevention, Atlanta, Georgia

KEY WORDS

American Indian, Alaska Native, immunization assessment, health disparities

\section{ABBREVIATIONS}

Al/AN-American Indian/Alaska Native

CHSDA — contract health services delivery areas

$\mathrm{Cl}$-confidence interval

$\mathrm{DTaP} \longrightarrow \geq$ Diphtheria and tetanus toxoids and pertussis vaccine, or diphtheria and tetanus toxoids, or diphtheria and tetanus toxoids and any acellular pertussis vaccine

Hib-Haemophilus influenzae type $b$

HepB-hepatitis B

IHS - Indian Health Service

I/T/U—Indian Health Service, Tribal, Urban Indian health facility

MMR-measles, mumps, rubella

MSA-Metropolitan Statistical Area

NIS-National Immunization Survey

PCV_- pneumococcal conjugate vaccine

Var-varicella

VPD—vaccine-preventable diseases

4:3:1:3:3:1— $\geq 4$ doses, diphtheria and tetanus toxoids and pertussis vaccine, diphtheria and tetanus toxoids, or diphtheria and tetanus toxoids and any pertussis vaccine, $\geq 3$ doses of oral or inactivated polio vaccine, $\geq 1$ dose of measles, mumps, and rubella vaccine, $\geq 3$ doses of Haemophilus influenzae type $b$ vaccine, $\geq 3$ doses of hepatitis $B$ vaccine, and $\geq 1$ dose of varicella vaccine $4: 3: 1: x: 3: 1$ - the $4: 3: 1: 3: 3: 1$ series excluding the Hib vaccine

4:3:1:3:3:1:4— $\geq 4$ doses diphtheria and tetanus toxoids and pertussis vaccine, diphtheria and tetanus toxoids, or diphtheria and tetanus toxoids and any pertussis vaccine, $\geq 3$ doses of oral or inactivated polio vaccine, $\geq 1$ dose of measles, mumps, and rubella vaccine, $\geq 3$ doses of Haemophilus influenzae type b vaccine, $\geq 3$ doses of hepatitis $B$ vaccine, $\geq 1$ dose of varicella vaccine, and $\geq 4$ doses of pneumococcal conjugate vaccine $4: 3: 1: x: 3: 1: 4$ — the $4: 3: 1: 3: 3: 1: 4$ series excluding the Hib vaccine

(Continued on last page) 
During the prevaccine era in the United States, the American Indian and Alaska Native (Al/AN) population suffered disproportionately from vaccinepreventable diseases (VPDs) such as Haemophilus influenzae type b (Hib), invasive pneumococcal disease, hepatitis $\mathrm{A}$, and hepatitis B. ${ }^{1}$ While routine childhood vaccination resulted in large declines in VPDs, maintaining high vaccination coverage is important, especially for populations at increased risk of disease. The Al/AN population remains vulnerable to VPDs due to risk factors such as higher background rates of disease, household crowding, and lack of running water in some Al/ AN communities..$^{1-3}$ Studies have also shown that many of the risk factors associated with underimmunization are more prevalent among Al/AN children compared with white children., ${ }^{4,5}$ A previous study on vaccination coverage in this population found that nationally, disparities in vaccine coverage between Al/AN and white children existed from 2001 through 2004 but were absent in 20054; a 2010 study in North Dakota, however, found that Al children were significantly less likely to be up to date with their vaccinations compared with white children. ${ }^{6}$

According to the 2010 census, the Al/AN population represents $\sim 1.7 \%$ of the US population?. Al/AN people receive health care from a variety of sources including the Indian Health Service (IHS) and private and/or public health insurance programs. ${ }^{8,9}$ The IHS is a federal health program for Al/AN people that provides care through a network of IHS, tribal, and urban Indian health facilities (I/T/U) to eligible Al/AN people. Approximately 2 million Al/AN people, $38 \%$ of the total US Al/AN population, are eligible to receive care at these predominantly rural $\mathrm{I} / \mathrm{T} / \mathrm{U}$ health facilities. ${ }^{10}$ However, some Al/ AN people who are eligible for care may not reside near an I/T/U facility, as the majority of $\mathrm{Al} / \mathrm{AN}$ people reside in urban areas. ${ }^{11,12}$ In addition, some Al/ AN people are not eligible to receive care from the I/T/U system because they are not enrolled members of a federally recognized tribe. While IHS monitors vaccination coverage among Al/AN children served by an I/T/U facility, ${ }^{13}$ it cannot produce national estimates of vaccination coverage for the Al/AN population.

The overall objective of this study was to describe vaccination coverage levels for Al/AN children in the United States between 2006 and 2010, examining whether the gains found for Al/AN children in $2005^{4}$ have been sustained.

\section{METHODS}

Data from the 2006 through the 2010 National Immunization Surveys (NIS) were analyzed. The NIS is an ongoing, national, random-digit-dial telephone survey of households with children 19 to 35 months old at the time of interview. The household telephone survey is followed by a survey mailed to the immunization providers identified during the telephone survey and for which permission was granted. All vaccination coverage estimates are based on provider-reported vaccinations. The household response rates for each year of the NIS studied (2006-2010) as defined by the Council of American Survey Research Organizations (CASRO) ${ }^{14}$ were $64.5 \%, 64.9 \%, 63.2 \%, 63.9 \%$, and $63.8 \%$, respectively. Adequate provider vaccination records were obtained for $70.4 \%$ of children with completed household interviews in 2006 ( $n=21044), 68.6 \%$ in $2007(n=17017), 71.0 \%$ in $2008(n=18$ 430), $70.7 \%$ in $2009(n=17313)$, and $71.2 \%$ in $2010(n=17004){ }^{15-19}$ Methodological details of the NIS have been previously published. ${ }^{20}$

\section{Vaccination Coverage Definitions}

Vaccination coverage assessed in this study included $\geq 4$ doses of diphtheria and tetanus toxoids and pertussis vaccine, or diphtheria and tetanus toxoids, or diphtheria and tetanus toxoids and any acellular pertussis vaccine $(4+\mathrm{DTaP}), \geq 3$ doses of poliovirus vaccine ( $3+$ polio), $\geq 1$ dose of measles, mumps, and rubella (1+MMR) vaccine, $\geq 3$ doses of Haemophilus influenza type $b(3+H i b)$ vaccine, $\geq 3$ doses of hepatitis $B(3+\mathrm{HepB})$ vaccine, $\geq 1$ dose of varicella $(1+\operatorname{Var})$ vaccine, and $\geq 4$ doses of pneumococcal conjugate $(4$ $+P C V)$ vaccine. In addition, we examined coverage with the combined 4 +DTaP, 3+polio, 1+MMR, 3+Hib, 3+HepB, and $1+\operatorname{Var}$ vaccine series, referred to as the 4:3:1:3:3:1 series, and coverage with this series plus 4+PCV, referred to as the $4: 3: 1: 3: 3: 1: 4$ series. Finally, because of the Hib vaccine shortage from 2007 to 2009,21 we analyzed coverage for these 2 series without Hib vaccine (4:3:1:x:3:1, 4:3:1:x:3:1:4).

\section{Race Variable Definition}

During the telephone portion of the NIS, the parent/guardian of the child was asked a series of questions about the race/ethnicity of the child. These included: "Is [child] of Hispanic or Latino origin?" and "Now, I am going to read a list of categories. Please choose one or more of the following categories to describe [child's] race. Is [child] white, black or African American, American Indian, Alaska native, Asian, native Hawaiian or other Pacific Islander?" By using responses to these questions, we defined white as all non-Hispanic children who were identified by their parents as white alone, and Al/AN as all non-Hispanic children who were identified by their parents as either Al/AN alone or Al/AN in combination with any other race. Hispanic children were excluded from the Al/AN definition as this designation may include American Indians from Central or South America. To determine if there were differences in coverage estimates depending on 
the population definition used for Al/ $\mathrm{AN}$, we compared vaccination coverage estimates for $\mathrm{Al} / \mathrm{AN}$ alone to $\mathrm{Al} / \mathrm{AN}$ in combination with another race. All results refer to the combined group of Al/AN alone or in combination with another race.

\section{Demographic Variable Definitions}

Demographic characteristics of the children in the sample were obtained during the telephone interview of the parent/guardian, as part of routine NIS data collection. Poverty level was determined by using household reported income level, number of people reported living in the household and US poverty thresholds. Characteristics of providers are provider-reported (ie, provider type). Three additional geographic variables were included: IHS Contract Health Service Delivery Areas, or CHSDAs, are defined by IHS and represent the catchment area for IHS-funded facilities. For regional comparisons, the IHS administrative areas were combined into 6 larger regions as has been done in previous studies. ${ }^{22}$ Lastly, Metropolitan Statistical Area (MSA) was used for urban versus rural comparisons.

\section{Statistical Methods}

The percentage of Al/AN children who were vaccinated was compared with the percentage of white children vaccinated by using Wald $\chi^{2}$ tests. Proportions are reported along with $95 \%$ confidence intervals (Cls). Data were weighted to adjust for households having multiple telephone lines, nonassessment of households without telephones, household unit nonresponse, provider nonresponse, and to reflect population demographic totals. A 2sided significance level of .05 was adopted for all statistical tests. Analysis were conducted by using SAS, release 9.2 (SAS, Cary, NC) and SUDAAN, release 10.0 (Research Triangle Institute, Re- search Triangle Park, NC) to take into account the complex nature of the survey.

\section{RESULTS}

There were no differences in vaccination coverage estimates between children who were Al/AN-only compared with Al/ $\mathrm{AN}$ in combination with another race with the one exception of $3+\mathrm{HepB}$ in 2008 ( $91.5 \%$ vs $98.6 \%, P=.01$ ). All subsequent Al/AN results refer to the combined group of Al/AN alone or in combination with another race. Al/AN sample sizes are included in Table 1, and ranged from 421 to 519 in any given year.

For all 5 years examined, Al/AN children were more likely than white children to live below the poverty level, have younger mothers, and have mothers with less education (Table 1). Mothers of Al/AN children were also less likely than mothers of white children to have ever been married. In 2006-2008, Al/AN children were significantly more likely to live in a household with more children; additional results are presented in Table 1.

Overall there were no significant differences in vaccination coverage between AI/AN and white children with the following vaccines in any of the 5 survey years: 4+DTaP, 3+polio, 3+Hib, or $3+$ HepB (Table 2 and Fig 1). In 2007, Al/ AN children had higher $1+$ Var vaccine coverage than white children $(92.8 \%$ vs $89.2 \%, P<.05)$, and in 2008, Al/AN children had higher 1+MMR coverage than white children $(95.3 \%$ vs $91.3 \%, P$ $<.01)$. Al/AN children had significantly lower 4+PCV coverage than white children in both 2008 (74.9\% vs $81.4 \%)$ and 2009 (71.4\% vs $83.4 \%$; both $P<$ .05); however, coverage did not differ in 2010. There were no significant differences between Al/AN and white children in the 4:3:1:3:3:1 vaccination series in any of the years studied. In 2006 and 2009, coverage with the 4:3:1:3:3:1:4 series was lower for Al/AN children ( $51.2 \%$ vs $62.7 \%$ in $2006 ; 52.3 \%$ vs $64.1 \%$ in 2009 , both $P<.05$ ) and in 2009 coverage with the 4:3:1:x:3:1:4 series was lower for Al/AN children (58.1\% vs $72.4 \%, P<.01$ ).

There were some differences in vaccination coverage levels between Al/AN children and white children in all 6 regions (Table 3). For the Northern Plains region in 2006, Al/AN children had lower coverage with 4+DTaP, 3+ polio, and $3+$ Hib vaccines compared with white children and lower 4:3:1: $x: 3: 1: 4$ coverage in 2009, although coverage in other years was not significantly different. Vaccination coverage levels for all other regions for all other years revealed that Al/AN children had either the same or higher coverage compared with white children (Table 3 ). For all years studied, between $63 \%$ and $69 \%$ of $\mathrm{Al} / \mathrm{AN}$ children resided in a CHSDA county. There were no significant differences in vaccine coverage among AI/AN children residing in a CHSDA county compared with Al/AN children not residing in a CHSDA county. There were few differences in coverage between Al/AN children residing in an MSA versus non-MSA; Coverage with 4:3:1:3:3 (2006), 3+polio (2007), and $1+$ MMR and $1+$ Var vaccines (2008) were higher among Al/AN children residing in a non-MSA compared with those residing in an MSA. There were no differences in coverage for 2009 or 2010.

\section{DISCUSSION}

From 2006 through 2010, few differences in vaccination coverage between Al/AN and white children were found. Indeed, in some regions (Southwest and Alaska), coverage for Al/AN children was frequently higher than that for white children. Instances of significantly lower coverage for Al/AN children were relatively rare, and by 2010 there was no evidence of vaccination coverage disparities between Al/AN children and white children. These 


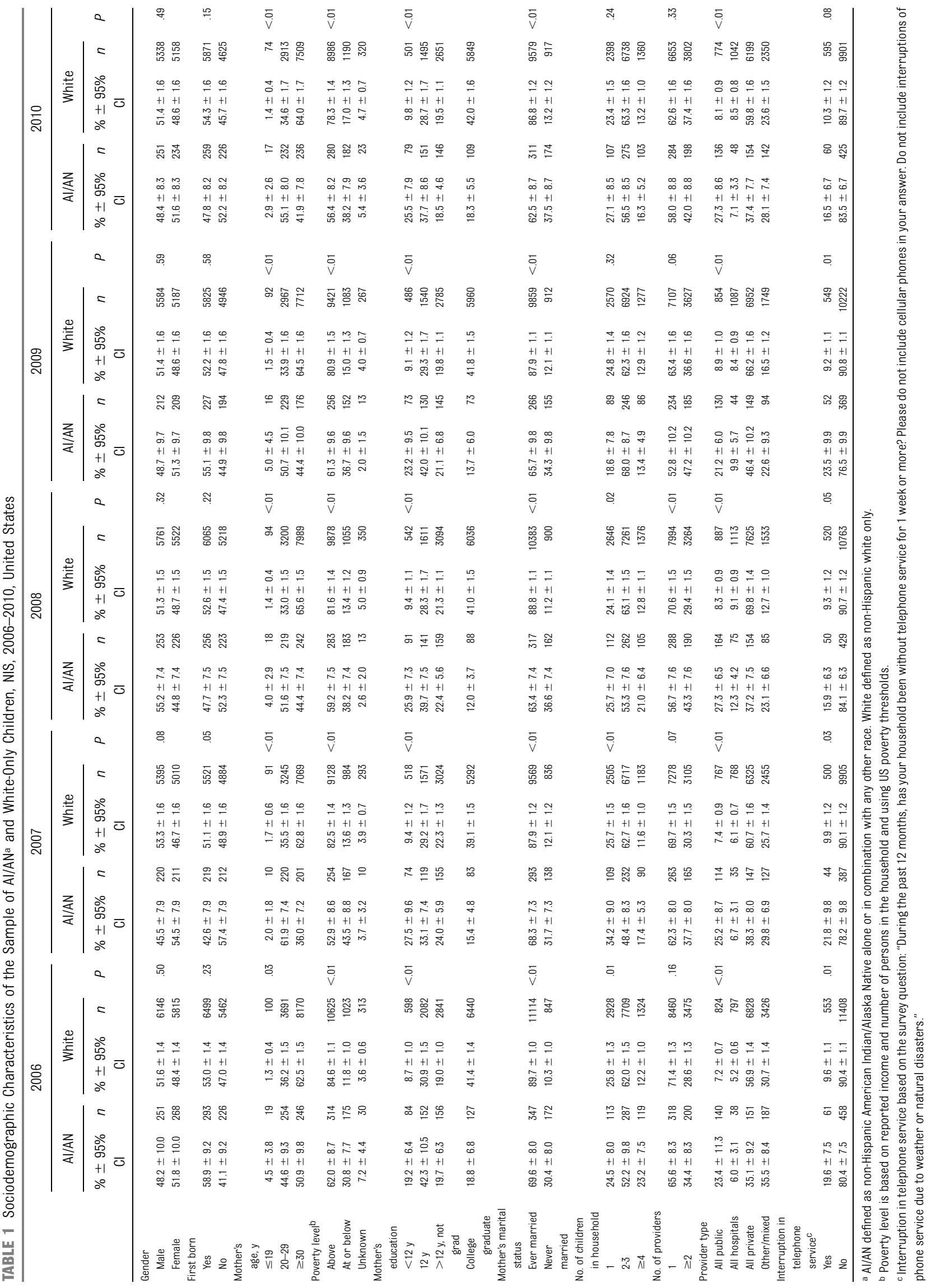




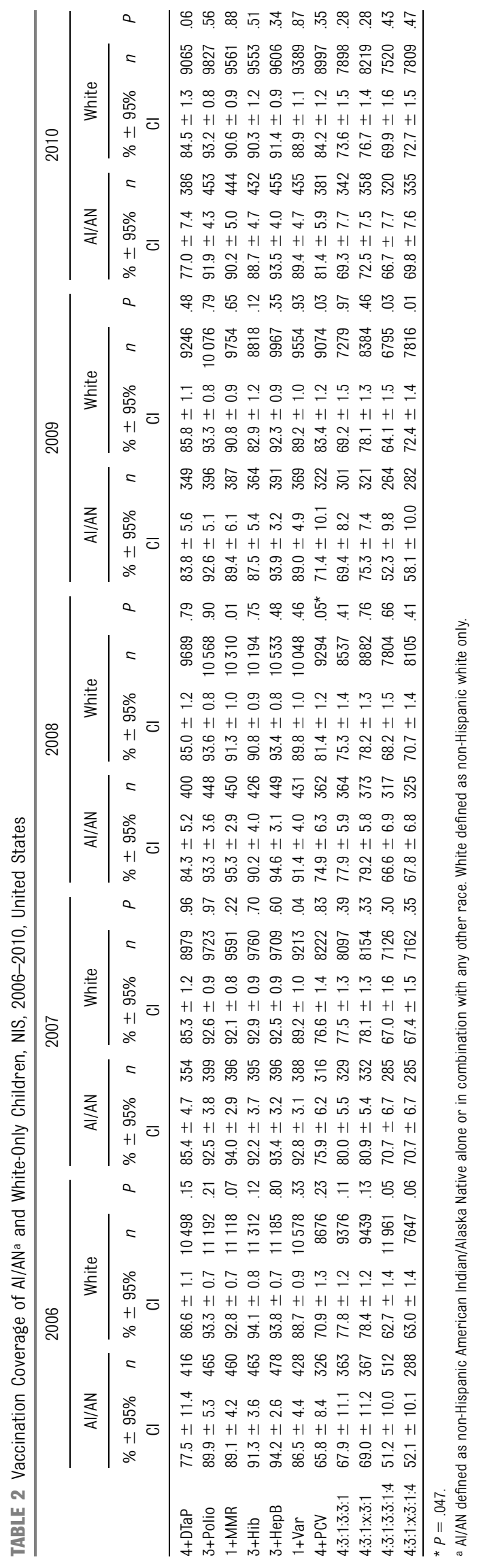

findings suggest that programs like the Vaccines for Children program that began in 1994, and provides free vaccine for Al/AN children younger than 19 years of age, ${ }^{23}$ as well as health care infrastructure improvements and vaccine delivery strategies used by $1 / T / U$ facilities, ${ }^{1,4,5}$ have been successful in improving immunization coverage for Al/AN children across the country.

Due to the 2008 and 2009 Hib vaccine shortage, the overall decrease in coverage with the Hib vaccine and the series containing Hib vaccine in those years is not unexpected. ${ }^{24}$ To ensure vaccine was provided to those at higher risk, the Advisory Committee on Immunization Practices (ACIP) recommended that Al/AN children continue to receive the full Hib series, and PedvaxHib vaccine from the CDC stockpile was made available for use in this population. ${ }^{25}$ In 2009, coverage with the 4:3:1:3:3:1 series nationally was higher for Al/AN children compared with white children. There was no difference in Hib vaccine coverage among Al/AN children compared with white children nationally, although 2 IHS regions (Alaska, Southwest) did achieve higher Hib coverage among Al/ AN children in 2008 and 2009. Both Alaska and the Southwest used PedvaxHib vaccine almost exclusively even before the shortage, whereas other regions used a mix of Hib products, which may explain why the higher Hib vaccine coverage was limited to these regions. Higher coverage with other vaccines in both these regions in 2008 and in Alaska in 2009, however, suggest that the Al/AN Hib recommendation was not the only contributing factor to the increased coverage reported among Al/AN children in these regions. Reasons for increased coverage in these 2 regions may be related to population characteristics such as geographical concentration (eg reservations/villages) with an I/T/U facility in close proximity 


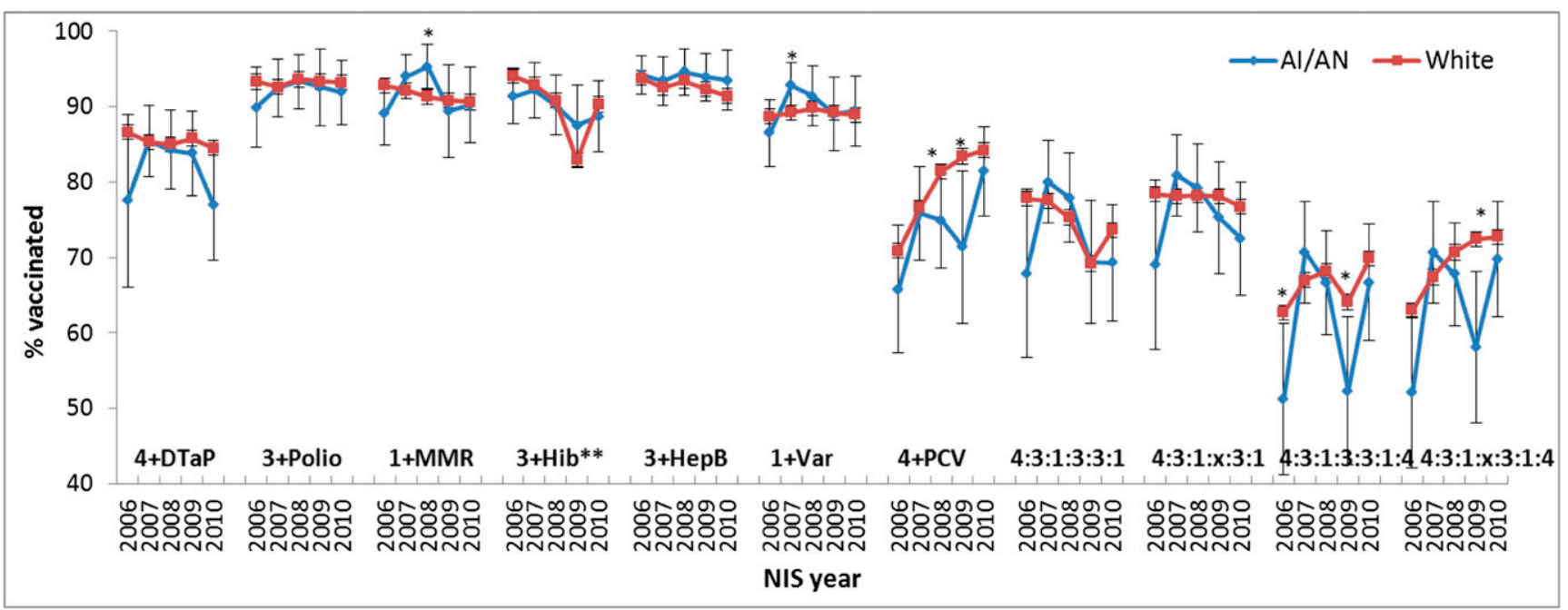

FIGURE 1

Vaccination coverage of AI/AN and white-only children, NIS, 2006-2010, United States. Al/AN defined as non-Hispanic American Indian/Alaska Native alone or in combination with any other race. White defined as non-Hispanic white only. ${ }^{\star} P<.05$ for comparison of Al/AN versus white children. ${ }^{\star \star} 3+$ Hib measure that does not take brand into account.

and the use of public health nurses to support immunization activities. ${ }^{1,4,5}$ In addition, higher immunization coverage among Alaska Natives compared with whites in Alaska has been noted in previous years ${ }^{5,26}$ and is likely a result of the collaborative efforts between state and tribal entities to deliver immunizations in remote Alaska Native villages.

Disparities present nationally in 2006 for the 4:3:1:3:3:1:4 series and in 2008 and 2009 for $4+$ doses of PCV (which also contributed to lower coverage with the 4:3:1:3:3:1:4 and 4:3:1:x:3:1:4 series in 2009) were not present in 2010. We could not determine if these differences were true differences or were due to random fluctuations in estimates from year to year. The sample size of Al/AN children is relatively small in the NIS in any one year, leading to large Cls around the estimates. Additionally, numerous statistical comparisons were made in this study with no correction made for multiple comparisons; therefore, some comparisons are likely to be statistically significant by chance alone. However, because the 4+PCV differences were found for 2 consecutive years, they may be more likely to have been true differences. It is unclear why coverage would have been lower for Al/AN children than for white children in 2008 and 2009, but it may be that coverage among Al/AN children was not increasing at the same rate as coverage among white children (Fig 1). Because certain Al/AN populations have an elevated rate of invasive pneumococcal disease compared with other populations, ${ }^{1}$ maintaining high PCV coverage for Al/AN children is important. In addition, while coverage at 2 years of age is helpful for monitoring disparities, looking at timeliness of vaccination before 2 years of age may provide additional insight. Future studies to examine the timeliness of vaccination in this population are needed.

Because the NIS is a random-digit-dial survey that samples at the proportion present in the population, the sample size of Al/AN children is relatively small each year. Other mechanisms for measuring vaccination coverage among the Al/AN population include the IHS data and state immunization registries. Vaccination coverage among Al/ AN children tracked by IHS is limited to those receiving care through $\mathrm{I} / \mathrm{T} / \mathrm{U}$ facilities. Immunization registries have the potential to provide more complete data on Al/AN children regardless of where they receive care and, as was done in North Dakota, ${ }^{6}$ can help to identify disparities at the local level that may not be present in national level estimates. Identifying local pockets of underimmunized children is important as regional differences exist and strategies to improve vaccination coverage for the Al/AN population may differ depending on where children are receiving care. Improving exchange of data between state immunization registries and $\mathrm{I} / \mathrm{T} / \mathrm{U}$ facilities could help ensure more complete data on the Al/ AN population to allow for these types of assessments.

This report's findings are subject to at least 5 limitations. First, before 2011, NIS was a landline telephone survey; although statistical weights adjust for nonresponse and nonlandline telephone households, as well as interruption in telephone service, some bias might remain. Second, although NIS relies on provider-verified vaccination histories, incomplete records and reporting could result in underestimates of coverage. Third, the exclusion of Hispanic Al/AN children may 
TABLE 3 Differences in Al/AN Versus White Non-Hispanic Vaccination Coverage by Regiona and by Year, NIS, 2006-2010, United States

\begin{tabular}{|c|c|c|c|c|c|c|c|c|c|c|c|c|}
\hline Region & Year & 4+DTaP & $3+$ Polio & $1+\mathrm{MMR}$ & $3+\mathrm{Hib}$ & $3+\mathrm{HepB}$ & $1+\operatorname{Var}$ & $4+\mathrm{PCV}$ & $4: 3: 1: 3: 3: 1$ & 4:3:1:x:3:1 & $4: 3: 1: 3: 3: 1: 4$ & 4:3:1:x:3:1:4 \\
\hline \multirow[t]{5}{*}{ East } & 2006 & - & - & - & - & - & - & - & - & - & - & - \\
\hline & 2007 & - & - & - & - & - & - & - & - & - & - & - \\
\hline & 2008 & - & - & - & - & - & - & - & - & - & - & - \\
\hline & 2009 & - & - & - & - & - & - & - & - & - & - & - \\
\hline & 2010 & - & $\uparrow H$ & - & - & - & - & - & - & - & - & - \\
\hline \multirow{5}{*}{ Southern Plains } & 2006 & - & $\uparrow \mathrm{H}$ & - & - & $\uparrow H$ & - & - & $\uparrow H$ & $\uparrow \mathrm{H}$ & - & - \\
\hline & 2007 & - & - & - & - & - & - & - & - & - & - & - \\
\hline & 2008 & - & - & $\uparrow H$ & - & - & - & - & - & - & - & - \\
\hline & 2009 & - & - & - & - & - & - & - & - & - & - & - \\
\hline & 2010 & - & - & - & - & - & - & - & - & - & - & - \\
\hline \multirow[t]{5}{*}{ Northern Plains } & 2006 & $\downarrow L$ & $\downarrow L$ & - & $\downarrow L$ & - & - & - & $\downarrow L$ & $\downarrow L$ & - & - \\
\hline & 2007 & - & - & - & - & - & $\uparrow H$ & - & - & - & - & - \\
\hline & 2008 & - & - & $\uparrow H$ & - & - & - & - & - & - & - & - \\
\hline & 2009 & - & - & - & - & - & - & - & - & - & - & $\downarrow L$ \\
\hline & 2010 & - & - & - & - & $\uparrow H$ & - & - & - & - & - & - \\
\hline \multirow[t]{5}{*}{ Southwest } & 2006 & - & - & - & - & - & - & - & - & - & - & - \\
\hline & 2007 & - & - & - & - & $\uparrow H$ & - & $\uparrow H$ & - & - & - & - \\
\hline & 2008 & $\uparrow H$ & $\uparrow H$ & $\uparrow H$ & $\uparrow H$ & $\uparrow H$ & - & - & $\uparrow H$ & $\uparrow H$ & - & - \\
\hline & 2009 & - & - & - & $\uparrow H$ & - & - & - & - & - & - & - \\
\hline & 2010 & - & - & - & - & - & - & - & - & - & - & - \\
\hline \multirow[t]{5}{*}{ Pacific Coast } & 2006 & - & - & - & - & - & - & - & - & - & - & - \\
\hline & 2007 & - & $\uparrow H$ & $\uparrow H$ & $\uparrow H$ & - & $\uparrow H$ & - & - & - & - & - \\
\hline & 2008 & - & $\uparrow \mathrm{H}$ & - & - & - & - & - & - & - & - & - \\
\hline & 2009 & - & - & - & - & - & - & - & - & - & - & - \\
\hline & 2010 & - & - & - & - & - & - & - & - & - & - & - \\
\hline \multirow[t]{5}{*}{ Alaska } & 2006 & $\uparrow H$ & - & - & - & - & - & $\uparrow H$ & $\uparrow H$ & $\uparrow H$ & $\uparrow H$ & $\uparrow H$ \\
\hline & 2007 & $\uparrow H$ & - & - & - & - & - & - & $\uparrow H$ & $\uparrow H$ & $\uparrow H$ & $\uparrow H$ \\
\hline & 2008 & $\uparrow \mathrm{H}$ & $\uparrow H$ & $\uparrow H$ & $\uparrow H$ & $\uparrow H$ & $\uparrow H$ & - & $\uparrow H$ & $\uparrow H$ & - & - \\
\hline & 2009 & - & - & $\uparrow H$ & $\uparrow H$ & - & $\uparrow H$ & - & $\uparrow H$ & $\uparrow H$ & - & - \\
\hline & 2010 & - & $\uparrow H$ & $\uparrow H$ & - & $\uparrow H$ & - & - & - & - & - & - \\
\hline
\end{tabular}

-, No statistically significant difference between Al/AN and white non-Hispanic; $\uparrow H$, Al/AN vaccination coverage statistically significantly $(P<.05)$ HIGHER than white non-Hispanic vaccination coverage; $\downarrow l$, Al/AN vaccination coverage statistically significantly $(P<.05)$ LOWER than white non-Hispanic vaccination coverage.

a The regions were defined by collapsing the IHS administrative regions into 6 regions. The definitions were as follows: East: ME, NH, VT, MA, RI, CT, NY, NJ, PA, MD, DE, OH, WV, VA, KY, NC, SC, TN MO, AR, LA, MS, AL, GA, FL; Southern Plains: KS, OK, TX; Northern Plains: MT, WY, ND, SD, NE, MN, IA, WI, IL, IN, MI; Southwest: NV, UT, AZ, NM, CO; Pacific Coast: WA, OR, ID, CA; Alaska; AK.

have produced some bias by excluding children of mixed Hispanic-Al/AN parents. Fourth, the sample size of Al/ AN children in any one year of the NIS is small, resulting in large Cls for the estimates. Because of the small sample size, there is much lower statistical power to detect small differences in vaccination coverage between Al/AN and other children, especially at sub- national levels. Last, a large number of statistical comparisons were made in this study and thus some were likely to be statistically significant by chance alone.

\section{CONCLUSIONS}

The gains in vaccination coverage found in 2005 by a previous study have been maintained. ${ }^{4}$ The absence of disparities in coverage with most vaccines between Al/AN children and white children from 2006 through 2010 is a clear success, although the ongoing presence of risk factors related to underimmunization and disparities in PCV coverage for 2 years highlights the need to continue these types of periodic reviews.

\section{REFERENCES}

1. Singleton R, Holve S, Groom A, et al. Impact of immunizations on the disease burden of American Indian and Alaska native children. Arch Pediatr Adolesc Med. 2009;163 (5):446-453

2. Gessner BD. Lack of piped water and sewage services is associated with pediatric lower respiratory tract infection in Alaska. J Pediatr. 2008;152(5):666670

3. Hennessy TW, Ritter T, Holman RC, et al. The relationship between in-home water service and the risk of respiratory tract, skin, and gastrointestinal tract infections among rural Alaska natives. Am J Public Health. 2008;98(11):2072-2078
4. Groom AV, Washington ML, Smith PJ, Bryan RT. Underimmunization of American Indian and Alaska Native children. Pediatrics. 2008;121(5):938-944

5. Strine TW, Mokdad AH, Barker LE, et al. Vaccination coverage of American Indian/ Alaska native children aged 19 to 35 months: findings from the National Immunization 
Survey, 1998-2000. Am J Public Health. 2003;93(12):2046-2049

6. LoMurray K, Sander M. Assessing vaccination coverage of American Indian children using the North Dakota immunization information system. Presented at the National Immunization Conference 2011, Atlanta, GA

7. Humes KR, Jones N, Ramirez R. Overview of race and Hispanic origin: 2010. U.S. Census. Available at: www.census.gov/prod/cen2010/ briefs/c2010br-02.pdf. Accessed 12/22/ 2010

8. Zuckerman S, Haley J, Roubideaux Y, LillieBlanton M. Health service access, use, and insurance coverage among American Indians/Alaska Natives and Whites: what role does the Indian Health Service play? Am J Public Health. 2004;94(1):53-59

9. Kaiser Family Foundation. Profile of American Indians and Alaska Natives and Their Health Coverage. 2009. Available at: www. kff.org/minorityhealth/7977.cfm. Accessed: $12 / 22 / 2011$

10. Indian Health Service. IHS fact sheets: IHS year 2011 profile. Available from: www.ihs. gov/PublicAffairs/IHSBrochure/Profile2011. asp. Accessed 11/14/2011

11. Castor ML, Smyser MS, Taualii MM, Park AN, Lawson SA, Forquera RA. A nationwide population-based study identifying health disparities between American Indians/Alaska Natives and the general populations living in select urban counties. Am J Public Health. 2006;96(8):1478-1484

12. Forquera R. Urban Indian Health. Kaiser Family Foundation Issue Brief. 2001. Available at: www.kff.org/minorityhealth/loader. cfm?url = /commonspot/security/getfile.cfm\& PagelD $=13909$. Accessed 12/22/2011

13. Indian Health Service. IHS Immunization Coverage Reports. Available from: www.ins. gov/epi/index.cfm?module = epi_vaccine_reports. Accessed 11/21/2011

14. Frankel L. The report of the CASRO Task Force on Response Rates. In: Wiseman F, ed. Improving data quality in sample surveys. Cambridge, MA: Marketing Science Institute; 1983

15. Centers for Disease Control and Prevention (CDC). National, state, and local area vaccination coverage among children aged 1935 months-United States, 2006. MMWR Morb Mortal Wkly Rep. 2007;56(34):880-885

16. Centers for Disease Control and Prevention (CDC). National, state, and local area vaccination coverage among children aged 1935 months-United States, 2007. MMWR Morb Mortal Wkly Rep. 2008;57 (35):961-966

17. Centers for Disease Control and Prevention (CDC). National, state, and local area vaccination coverage among children aged 1935 months-United States, 2008. MMWR Morb Mortal Wkly Rep. 2009;58(33):921-926

18. Centers for Disease Control and Prevention (CDC). National, state, and local area vaccination coverage among children aged 1935 months-United States, 2009. MMWR Morb Mortal Wkly Rep. 2010;59(36):11711177

19. Centers for Disease Control and Prevention (CDC). National and state vaccination coverage among children aged 19-35 monthsUnited States, 2010. MMWR Morb Mortal Wkly Rep. 2011;60(34):1157-1163
20. Smith P, Hoaglin D., Battaglia M., et al. Statistical methodology of the National Immunization Survey, 1994-2002.Vital Health Stat. 2005;2(138)

21. Centers for Disease Control and Prevention (CDC). Changes in measurement of Haemophilus influenzae serotype b (Hib) vaccination coverage - National Immunization Survey, United States, 2009. MMWR Morb Mortal Wkly Rep. 2010;59(33):1069-1072

22. Espey DK, Wiggins CL, Jim MA, Miller BA, Johnson CJ, Becker TM. Methods for improving cancer surveillance data in American Indian and Alaska Native populations Cancer. 2008;113(5 Suppl):1120-1130

23. Centers for Disease Control and Prevention. Vaccines for Children Program. Available from: www.cdc.gov/vaccines/programs/vfc/ default.htm. Accessed 11/21/2011

24. Santibanez TA, Shefer A, Briere EC, Cohn AC, Groom AV. Effects of a nationwide Hib vaccine shortage on vaccination coverage in the United States. Vaccine. 2012;30(5):941947

25. Centers for Disease Control and Prevention (CDC). Interim recommendations for the use of Haemophilus influenzae type $b$ (Hib) conjugate vaccines related to the recall of certain lots of Hib-containing vaccines (PedvaxHIB and Comvax). MMWR Morb Mortal Wkly Rep. 2007;56(50):1318-1320

26. Centers for Disease Control and Prevention (CDC). Vaccination coverage levels among Alaska Native children aged 19-35 monthsNational Immunization Survey, United States, 2000-2001. MMWR Morb Mortal Wkly Rep. 2003;52(30):710-713

(Continued from first page)

Ms Groom contributed substantially to the conception and design of the study and interpretation of data. She was the lead in drafting and revising the article. She approved submission of the article for publication. Dr Santibanez contributed substantially to the conception, design, and interpretation of data. She conducted the data analysis for this article and contributed substantially to the drafting and revision of the manuscript. She approved submission of this article for publication. Dr Bryan contributed substantially to the conception, design, and interpretation of data; provided critical review of the article; and participated in revisions. He approved submission of this article for publication.

www.pediatrics.org/cgi/doi/10.1542/peds.2012-1001

doi:10.1542/peds.2012-1001

Accepted for publication Jul 30, 2012

Address correspondence to Amy Groom, IHS Division of Epidemiology and Disease Prevention, 5300 Homestead Rd NE, Albuquerque, NM 87110. E-mail: Amy. Groom@ihs.gov

PEDIATRICS (ISSN Numbers: Print, 0031-4005; Online, 1098-4275).

Copyright (C) 2012 by the American Academy of Pediatrics

FINANCIAL DISCLOSURE: The authors have indicated they have no financial relationships relevant to this article to disclose.

FUNDING: No external funding. 